\title{
Cerebral time-domain near-infrared spectroscopy for clinical use
}

\author{
Lange, Frédéric
}

Frédéric Lange, "Cerebral time-domain near-infrared spectroscopy for clinical use," Proc. SPIE 11879, Frontiers in Biophotonics and Imaging, 1187907 (6 October 2021); doi: 10.1117/12.2604718

SPIE. Event: SPIE Photonex, 2021, Glasgow, Scotland, United Kingdom 


\title{
Cerebral time-domain near-infrared spectroscopy for clinical use
}

\author{
Frédéric Lange $\mathrm{a}^{\mathrm{a}^{*}}$ \\ ${ }^{a}$ Department of Medical Physics and Biomedical Engineering, University College London, UK \\ f.lange@ucl.ac.uk
}

\begin{abstract}
Near-infrared spectroscopy (NIRS) is an optical technique that can measure brain tissue oxygenation and haemodynamics in real time and at the patient bedside allowing medical doctors to access important physiological information. In particular, time-domain NIRS (or TD-NIRS) is the most advanced NIRS technique, collecting the biggest amount of information, increasing the accuracy of the measurements and enabling to extract detailed information of the absolute optical properties of the tissues. All these optical information allows to extract detailed physiological information about the brain, and also get some insights into to the tissue's anatomy. After presenting the basics of TDNIRS, typical applications of TD-NIRS in a clinical context will be presented.
\end{abstract}

Keywords: NIRS, Time-domain, Brain, fNIRS, Monte-Carlo, Clinical application

\section{PRINCIPLES OF TIME DOMAIN NIRS}

Near-infrared Spectroscopy (NIRS) is a non-invasive technique able to provide information about biological tissues noninvasively. NIRS relies on the fact that light in the biological window, i.e., between 600 and $1000 \mathrm{~nm}$, can penetrate deep into the biological tissues, because in that wavelength range absorption is low, and scattering is the dominant interaction process. Moreover, as the haemoglobin, in its oxygenated and deoxygenated form, is one of the dominant contributors to the light absorption, information about deep tissue oxygenation can be collected. This capacity has been largely used in order to extract information of several tissue types like muscle, breast or brain [1]. In particular, NIRS has been extensively used in order to gather information about the brain hemodynamic both in healthy people and in the clinic. Time-domain NIRS (TD-NIRS) is the most advanced form of NIRS, able to gather the largest amount of information. Basically, the principle of TD-NIRS is to emit a short pulse (i.e., ps) of coherent light at a point on the tissue and to record the distribution of the time-of-flight of photons (DTOF) several $\mathrm{mm}$ or $\mathrm{cm}$ away from the source point. This is an advantage over conventional NIRS, i.e., continuous waves NIRS (CW-NIRS), which only records a change in the light intensity at a detector. The time-of-flight information gathered with TD-NIRS expands the capacities of CW-NIRS with the main advantages of i) enabling to extract absolute information about the absorption and scattering parameters of the tissues that are then used to extract absolute concentration of chromophores in the tissues, allowing to compared different patient groups or individuals in the clinic, ii) enhancing the depth sensitivity of the measurement, as the time-of-flights encodes the penetration depth of photons, which is very useful especially for brain monitoring and iii) making the measurements less sensitive to signal fluctuation which makes it more robust in a clinical setting.

\section{TD-NIRS FOR BRAIN MONITORING IN A CLINICAL SETTING}

The numerous capacities of TD-NIRS make it a good candidate for clinical applications. Various applications were explored with great success like the monitoring of breast [2] or thyroid [3] tissues for oncological applications and, the most widespread one, brain monitoring. We will review bellow a few examples highlighting the strength of the use of TD-NIRS in a clinical context for brain monitoring. A more detailed review of these applications can be found in reference [4].

\subsection{Monitoring in the clinical environment}

Intensive Care Unit (ICU) is one of the most complicated environments to deploy a monitoring instrument as it is a busy place where the space is limited. Therefore, the instrument developed in order to cope with this special environment must be compact and easily operable. Several TD-NIRS instruments have been successfully used in the ICU for various pathologies. One of the main applications of TD-NIRS in the ICU is the monitoring of stroke patients. The utility of

Frontiers in Biophotonics and Imaging, edited by Sumeet Mahajan, Stefanie Reichelt, Proc. of SPIE

Vol. 11879, $1187907 \cdot$ @ 2021 SPIE · CCC code: 0277-786X/21/\$21 · doi: 10.1117/12.2604718 
using NIRS in the context of stroke has been reviewed in [5] and the authors concluded that using TD-NIRS is mandatory for the assessment of pathophysiological state-dependent variables such as perfusion or absolute values of tissue oxygenation. We can cite the study by Giacalone and colleagues [6] that used a TD-NIRS instrument to measure haemoglobin concentrations and oxygen brain tissue saturation $\left(\mathrm{StO}_{2}\right)$ of the anterior circulation in stroke patients. In this study, 47 stroke patients were monitored in the stroke unit at the bedside. Multiple areas of the brain were probed (i.e., frontal, parietal, occipital) and CT-scans images were used to categorize brain regions below each TD-NIRS probe. Moreover, the results were compared with a control group selected from a previous study [7]. Significant differences of haemoglobin concentrations and $\mathrm{StO}_{2}$ were found between patients and controls, and among patients according to recanalization status. This demonstrate the strength of TD-NIRS that is able to be operated in the ICU and extract absolute values of hemodynamic parameters that can be used to compare several individuals objectively.

Another application of TD-NIRS in the ICU is the monitoring of traumatic brain injuries (TBI) patients [8]. Highton and colleagues [9] focused on the changes in light scattering related to TBI. In this study, a total of 21 TBI patients were monitored with a TD-NIRS system. In 10 patients suffering from unilateral infarction, even though no significant difference in $\mathrm{StO}_{2}$ or haemoglobin concentration were found, a significant reduction of $\mu$ ', the reduced scattering coefficient that is related to the tissue structure, was related to the injury severity. This highlight another strength of TDNIRS which is the ability to collect structural information in complement to the hemodynamic parameters. In a clinical context, where the anatomy of the brain is likely to be affected by the patient's condition, especially in TBI, this extra information available at the bedside without the need of more complex imagery like MRI, can be extremely valuable.

Finally, another complex setting to deploy a monitoring instrument is the operating room. The same constraints as in the ICU apply with the need of a compact and robust system in order to avoid any disturbance to the surgical procedure. The use of TD-NIRS in the peroperative context has also been reported. For example, Ohmae and colleagues [10] reported the monitoring of cerebral circulation during cardiopulmonary bypass surgery using a TD-NIRS instrument. In this study, the brain oxygenation of 23 patients who underwent coronary-artery bypass surgery were measured. The authors reported a large fluctuation of the mean optical pathlength (MOP) among patients. In the case of a typical CW-NIRS measurement, the MOP cannot be measured and is taken from tabulated values. Moreover, it is often considered constant over the course of the measurement. The reported large fluctuation of the MOP highlights the importance of the absolute monitoring enabled by TD-NIRS, in order to produce accurate monitoring in environment were the typical NIRS assumptions (for example constant MOP) do not hold.

\subsection{Improving the understanding of the pathophysiology}

On top of the monitoring of patients at bedside, TD-NIRS can be used to better understand the underlying pathophysiology of diseases. For example, our group has reported a feasibility study on the use of TD-NIRS to monitor the cerebral oxygenation of people with multiple sclerosis (pwMS) [11]. In this work, we use our in-house TD-NIRS system [12] to monitor the baseline brain oxygenation and the changes in haemodynamics and oxygenation following a functional stimulation of a cohort of pwMS. This study demonstrated the capacity of our system to be operated in this particular population. One of the strengths of this study is that our subjects were recruited from an ongoing phase 3 clinical trial (MS-STAT 2) that explores the effect of simvastatin for secondary progressive MS. Therefore, on top of the optical data, we have access to a unique clinical dataset including anatomical MRI, cognitive evaluations and fluid biomarkers. This unique dataset allows us to explore in detail our TD-NIRS recording. For example, we are able to use the MRI scans of our participant to study the light propagation in the MS brain, via Monte-Carlo simulation of the light transport. Indeed, one of the particularities of MS is that it induces a cerebral atrophy, which modifies the light propagations in the tissues. This particularity then needs to be taken into account during the data processing to reduce undesirable effects which could prevent an accurate comparison between subjects. Therefore, having access to this detailed dataset can help use to better understand the output of the TD-NIRS instruments in order to optimize their operation, even in non-clinical studies, where such detailed information might not be available.

\section{CONCLUSIONS}

TD-NIRS has been proven to be a valuable tool to provide information about the brain haemodynamic and structure in a clinical environment. From a research perspective, the use of TD-NIRS in a clinical context can also benefit the developers, as more information can be obtained, like MRI scans or more refined physiological monitoring. This extra information can then be used to refine both the instruments and the data processing methods used in order to increase the accuracy of the technique, which in turns will push even further the adoption of TD-NIRS by clinicians. 


\section{REFERENCES}

[1] C. E. Elwell and C. E. Cooper, "Making light work: illuminating the future of biomedical optics.," Philos. Trans. A. Math. Phys. Eng. Sci., vol. 369, no. 1955, pp. 4358-79, Nov. 2011, doi: 10.1098/rsta.2011.0302.

[2] S. Vedantham and A. Karellas, "Emerging Breast Imaging Technologies on the Horizon," Semin. Ultrasound, CT MRI, vol. 39, no. 1, pp. 114-121, 2017, doi: 10.1053/j.sult.2017.09.004.

[3] L. Cortese et al., "The LUCA device: a multi-modal platform combining diffuse optics and ultrasound imaging for thyroid cancer screening," Biomed. Opt. Express, vol. 12, no. 6, p. 3392, 2021, doi: 10.1364/boe.416561.

[4] F. Lange and I. Tachtsidis, "Clinical Brain Monitoring with Time Domain NIRS: A Review and Future Perspectives," Appl. Sci., vol. 9, no. 8, p. 1612, Apr. 2019, doi: 10.3390/app9081612.

[5] H. Obrig and J. Steinbrink, "Non-invasive optical imaging of stroke," Philos. Trans. R. Soc. A Math. Phys. Eng. Sci., vol. 369, no. 1955, pp. 4470-4494, 2011, doi: 10.1098/rsta.2011.0252.

[6] G. Giacalone et al., "Time-domain near-infrared spectroscopy in acute ischemic stroke patients," Neurophotonics, vol. 6, no. 01, p. 1, 2019, doi: 10.1117/1.NPh.6.1.015003.

[7] G. Giacalone et al., "Cerebral time domain-NIRS: reproducibility analysis, optical properties, hemoglobin species and tissue oxygen saturation in a cohort of adult subjects," Biomed. Opt. Express, vol. 8, no. 11, p. 4987, Nov. 2017, doi: 10.1364/boe.8.004987.

[8] D. J. Davies et al., "Near-Infrared Spectroscopy in the Monitoring of Adult Traumatic Brain Injury: A Review," J. Neurotrauma, vol. 32, no. 13, pp. 933-941, Jul. 2015, doi: 10.1089/neu.2014.3748.

[9] D. Highton, I. Tachtsidis, A. Tucker, C. Elwell, and M. Smith, Near Infrared Light Scattering Changes Following Acute Brain Injury, vol. 876. New York, NY: Springer New York, 2016.

[10] E. Ohmae et al., "Clinical evaluation of time-resolved spectroscopy by measuring cerebral hemodynamics during cardiopulmonary bypass surgery," J. Biomed. Opt., vol. 12, no. 6, p. 062112, 2007, doi: 10.1117/1.2804931.

[11] F. Lange, T. Williams, J. Chataway, K. J. Smith, and I. Tachtsidis, "Cerebral Time Domain Near-infrared Spectroscopy of people with Multiple Sclerosis: a feasibility study," in European Conferences on Biomedical

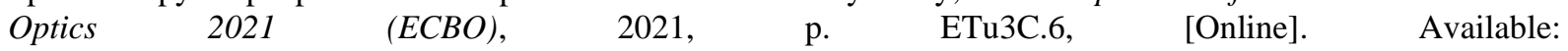
http://www.osapublishing.org/abstract.cfm?URI=ECBO-2021-ETu3C.6.

[12] F. Lange, L. Dunne, L. Hale, and I. Tachtsidis, "MAESTROS: A Multiwavelength Time-Domain NIRS System to Monitor Changes in Oxygenation and Oxidation State of Cytochrome-C-Oxidase," IEEE J. Sel. Top. Quantum Electron., vol. 25, no. 1, pp. 1-12, Jan. 2019, doi: 10.1109/JSTQE.2018.2833205. 